SUHER CAROLINA YABROUDI BAYRAM

REMOÇÃO DE MATÉRIA ORGÂNICA E NITROGÊNIO DE LIXIVIADOS DE ATERRO SANITÁRIO. TRATAMENTO POR NITRITAÇÃO/DESNITRITAÇÃO BIOLÓGICA E PROCESSOS FÍSICO-QUÍMICOS 


\title{
REMOÇÃO DE MATÉRIA ORGÂNICA E NITROGÊNIO DE LIXIVIADOS DE ATERRO SANITÁRIO. TRATAMENTO POR NITRITAÇÃO/DESNITRITAÇÃO BIOLÓGICA E PROCESSOS FÍSICO-QUÍMICOS
}

\author{
Tese apresentada à Escola Politécnica da \\ Universidade de São Paulo para obtenção do \\ título de Doutor em Engenharia.
}

Área de concentração:

Engenharia Hidráulica e Sanitária

Orientador:

Pedro Alem Sobrinho 
Este exemplar foi revisado e corrigido em relação à versão original, sob responsabilidade única do autor e com a anuência de seu orientador.

São Paulo, de março de 2013.

Assinatura do autor

Assinatura do orientador

Yabroudi Bayram, Suher Carolina

Remoção de matéria orgânica e nitrogênio de lixiviados de aterro sanitário: tratamento por nitritação/desnitritação biológica e processos físico-químicos / S.C. Yabroudi Bayram. -versão corr. -- São Paulo, 2012.

$192 \mathrm{p}$.

Tese (Doutorado) - Escola Politécnica da Universidade de São Paulo. Departamento de Engenharia Hidráulica e Ambiental.

1. Nitrogênio 2. Nitrificação 3. Resíduos sólidos I. Universidade de São Paulo. Escola Politécnica. Departamento de Engenharia Hidráulica e Ambiental II. t. 
À memória de meu querido pai, Jamal Yabroudi, seu exemplo de vida, valores, princípios e amor incondicional à sua família serão hoje e sempre meu maior tesouro.

À minha mãe Fadwa de Yabroudi, por seu apoio e palavras de carinho nos momentos mais difíceis. 


\section{AGRADECIMENTOS}

Ao Prof. Dr. Pedro Alem Sobrinho pela orientação, conselhos e paciência nos momentos cruciais deste trabalho. Obrigada por ter confiado na minha capacidade, embora muitas vezes não tenha atendido suas expectativas.

À Prof ${ }^{a}$. Dra. Dione Mari Morita, quem além de ter me orientado e compartilhado longas horas de trabalho, contribuindo com seus valiosos conhecimentos a esta pesquisa, me brindou com sua amizade e palavras de conforto nos momentos mais difíceis. Sem seu apoio constante, teria sido impossível concluir o trabalho.

Ao Prof. Dr. Roque Passos Piveli, pelas sugestões, apoio, carinho e amizade.

Ao Prof. Dr. Sidney Seckler Ferreira Filho, pela ajuda nos momentos precisos.

Aos funcionários do Laboratório de Saneamento Prof. Lucas Nogueira Garcez: Fábio, Campos e Laerte Carvalho, pela grande ajuda e apoio nas análises e, principalmente, pela amizade que permanece.

Aos Professores da Universidade de Zulia (Maracaibo - Venezuela), Carmen Cárdenas, Zullay Mármol, Ismenia Araujo, Luis Vargas, Antonio De Turris, Caterina Aiello e Virginia Pacheco, pela paciência e apoio nesta etapa da minha vida.

Aos colegas de pós-graduação e amigos Alan, Luciano, Mailer, Humberto, Gilberto, Rodrigo Weverton e Márcia, pelo apoio, carinho e momentos de alegria e convivência prazerosa dentro e fora do laboratório.

À Mari Nishimura e Marli Meireles, pela amizade, conselhos, sugestões e por ter me feito sentir como outro membro das suas famílias.

Aos funcionários da estação de recepção de efluentes Piqueri da SABESP, pela ajuda na coleta das amostras de lixiviado.

Aos funcionários do Departamento de Engenharia Hidráulica e Sanitária da EPUSP, Ângela Mizuta; Wándrea Dantas; Odorico Borges e Ricardo Souza, pelo ajuda, orientações e carinho dispensado. 
Yabroudi, S.C. Remoção de matéria orgânica e nitrogênio de lixiviados de aterro sanitário. Tratamento por nitritação/desnitritação biológica e processos físico-químicos. 192 p. Tese (Doutorado). Escola Politécnica da Universidade de São Paulo. São Paulo, 2012.

\section{RESUMO}

A presente pesquisa objetivou estudar a remoção de matéria orgânica e nitrogênio de lixiviados de aterro sanitário, através do processo biológico de nitritação/desnitritação, operando um reator de lodos ativados em bateladas sequenciais e pós-tratamento físico químico. O trabalho foi dividido em cinco etapas. Durante a primeira, buscou-se ajustar a duração das etapas anóxica e aerada e as condições operacionais, assim como avaliar a utilização da matéria orgânica presente no lixiviado como fonte de carbono. Ao longo de 16 ciclos de tratamento, os resultados da etapa anóxica indicaram que a duração desta não deveria ser maior que uma hora. A desnitritação ficou limitada ao baixo conteúdo de matéria orgânica de fácil biodegradação no lixiviado, o que levou a baixas taxas, entre 0,010 e 0,142 $\mathrm{kg} . \mathrm{N}-\mathrm{NO}_{2}{ }^{-} / \mathrm{m}^{3}$.dia. Na segunda etapa, foi ajustada a duração da reação anóxica em uma hora, registrando taxa de desnitritação volumétrica média de $1,704 \mathrm{~kg} . \mathrm{N}-\mathrm{NO}_{2}{ }^{-} / \mathrm{m}^{3}$.dia, assim como remoção de $80 \%$ da DBO e $47 \%$ de COT, o que indica que a maior parte da matéria orgânica biodegradável foi empregada pelos micro-organismos heterotróficos na desnitritação. Ao final da fase aeróbia, ao longo da primeira e segunda etapa, mantendo uma concentração de oxigênio dissolvido na massa de líquido do reator de $2,0 \mathrm{mg} \cdot \mathrm{O}_{2} / \mathrm{L}$, foi possível alcançar eficiências de remoção de $\mathrm{N}-\mathrm{NH}_{3}$ de $98 \%$ com predominância do $\mathrm{N}-\mathrm{NO}_{2}{ }^{-}$, evidenciado pela relação $\mathrm{N}-\mathrm{NO}_{2}{ }^{-} /\left(\mathrm{N}-\mathrm{NO}_{2}{ }^{-}+\mathrm{N}^{-} \mathrm{NO}_{3}{ }^{-}\right)$próximo de 1 . As taxas volumétrias de nitritação variaram na faixa de 0,095 e $0,199 \mathrm{~kg} \cdot \mathrm{N}_{-} \mathrm{NH}_{3} / \mathrm{m}^{3}$.dia. Como o sobrenadante do reator principal apresentava concentrações elevadas de $\mathrm{N}^{-\mathrm{NO}_{2}}{ }^{-}$ao final da fase aeróbia, o efluente foi aplicado em um reator de polimento adicionou-se etanol como fonte de carbono. $\mathrm{O}$ tratamento complementar ocorreu sem desequilíbrios, apesar do prolongado tempo de reação anóxica requerido para reduzir toda a massa de nitrito introduzida no sistema, fato que levou a registrar baixas taxas de desnitritação, entre 0,221 e $0,052{\mathrm{~kg} \mathrm{~N}-\mathrm{NO}_{2}}^{-} / \mathrm{kg} \mathrm{SSV}^{-d i a}$. Durante a quarta etapa, foi avaliado em um segundo reator de lodo ativado em bateladas sequenciais o processo de nitritação/desnitritação, adicionando dentro do reator, ao início da fase anóxica, o etanol para a redução biológica de todo o nitrogênio oxidado a nitrogênio gasoso. Com concentrações de OD no conteúdo do reator de $2,0 \mathrm{mg}$. $\mathrm{O}_{2} / \mathrm{L}$ e valores de $\mathrm{pH}$ de $8,27 \pm 0,27$, a relação $\mathrm{N}-\mathrm{NO}_{2}{ }^{-} /\left(\mathrm{N}^{-\mathrm{NO}_{2}}{ }^{-}+\mathrm{N}^{-\mathrm{NO}_{3}}{ }^{-}\right)$não foi superior a $70 \%$ nos 9 ciclos estudados, indicando a presença de nitrato. A hipótese que melhor explica a situação refere-se à possibilidade de que a biomassa esteja se adaptando as novas condições operacionais e a probabilidade de que a adição do etanol durante a reação anóxica possa estar criando condições que favorecem a atividade das bactérias oxidantes de nitrito, assim como os menores diâmetros de floco. A quinta etapa compreendeu a aplicação de um pós-tratamento com redução do $\mathrm{pH}$, adição de sais de ferro e carvão ativado em pó (CAP). Nestes testes, foram registradas remoções de COT e cor de de $93 \%$ e $98 \%$ respectivamente, mantendo $\mathrm{pH}$ em 3,0 e dosagens de 300 $\mathrm{mg} . \mathrm{Fe}^{+3} / \mathrm{L}$ e 15 g.CAP/L.

Palavras-chaves: Lixiviado. Nitrito, Nitritação. Desnitritação. Etanol. 
Yabroudi, S.C. Removal of organic matter and nitrogen from landfill leachate. Treatment by nitritation/denitritation biological and physico-chemical processes. $192 \mathrm{p}$. Thesis. Escola Politécnica da Universidade de São Paulo. São Paulo, 2012.

\begin{abstract}
This research aimed to study the removal of organic matter and nitrogen from landfill leachate through biological process of nitritation/denitritation, operating an activated sludge reactor in sequential batches, and physical- chemical post treatment. The work was divided into five stages. During the first stage, was tried to adjust the duration of the anoxic and aerated stages and operational conditions, and evaluate the use of organic matter present in the leachate as a carbon source. Over 16 cycles of treatment, the results of the anoxic stage indicated that this duration should not be greater than one hour. The denitritation was limited by the low organic matter rapidly biodegradable content into the leachate, which led to low rates between 0.010 and $0.142 \mathrm{~kg} . \mathrm{N}-\mathrm{NO}_{2}{ }^{-} / \mathrm{m}^{3}$.day. During the second stage, was adjusted the duration of the anoxic reaction to one hour, and showed medium volumetric denitritation rate of $1.704 \mathrm{~kg} . \mathrm{N}-$ $\mathrm{NO}_{2}{ }^{-} / \mathrm{m}^{3}$.day, as well as removal of $80 \%$ of BOD and $47 \%$ of TOC. These results indicate, most of the biodegradable organic matter was used for heterotrophic microorganisms for denitritation. At the end of aerobic phase, during the first and second stage, and maintaining a concentration of oxygen dissolved into the liquid mass of the reactor in $2.0 \mathrm{mg} . \mathrm{O}_{2} / \mathrm{L}$, it was possible to achieve removal efficiencies of $\mathrm{NH}_{3}-\mathrm{N}$ to $98 \%$ with predominance of $\mathrm{N}^{-\mathrm{NO}_{2}}{ }^{-}$, that was evidenced by the ratio $\mathrm{N}-\mathrm{NO}_{2}{ }^{-} /\left(\mathrm{N}_{-} \mathrm{NO}_{2}{ }^{-}+\mathrm{N}^{-} \mathrm{NO}_{3}{ }^{-}\right)$close to 1 . The volumetric nitritation rates varied from 0,095 to $0,199 \mathrm{~kg} \cdot \mathrm{N}-\mathrm{NH}_{3} / \mathrm{m}^{3}$.day. The effluent from the principal reactor contained high concentrations of $\mathrm{N}^{-\mathrm{NO}_{2}}{ }^{-}$at the end of the aerobic phase, for that reason it was applied a polishing stage where was added ethanol as a carbon source. Complementary treatment transcurred without problems, just was required a prolonged anoxic reaction to reduce the entire mass of nitrite introduced into the system, fact that lead to register low rates of denitritation, between 0.221 and $0.052 \mathrm{~kg} \mathrm{~N}-\mathrm{NO}_{2}{ }^{-} / \mathrm{kg}$ SSV.day. During the fourth stage, was evaluated in a second activated sludge sequential batch reactor; the nitritation/desnitritation process added ethanol inside the reactor, at the beginning of the anoxic phase, for the biological reduction from nitrogen oxidized to nitrogen gaseous. Maintained OD concentrations into the reactor of $2,0 \mathrm{mg} . \mathrm{O}_{2} / \mathrm{L}$ and $\mathrm{pH}$ values of $8.27 \pm 0.27$, the $\mathrm{N}-\mathrm{NO}_{2}{ }^{-} /\left(\mathrm{N}_{-} \mathrm{NO}_{2}{ }^{-}+\mathrm{N}^{-} \mathrm{NO}_{3}{ }^{-}\right)$ratio was not higher than $70 \%$ during the 9 cycles studied, indicating the presence of nitrate. The best hypothesis which explains this situation, related to the possibility of the biomass was adapted to the new operating conditions, and the possibility to addition ethanol at the begging of anoxic reaction, may be created adequate conditions for oxidizer nitrite bacteria, as well as the small floc diameters. The fifth stage consisted to apply a physical-chemical post-treatment with $\mathrm{pH}$ and addition of iron salts and powdered activated carbon (PAC). During these tests, was removal $93 \%$ and $98 \%$ of TOC and color respectively, with $\mathrm{pH}$ to 3,0 and doses of $300 \mathrm{mg} \cdot \mathrm{Fe}^{+3} / \mathrm{L}$ and $15 \mathrm{~g} . \mathrm{PAC} / \mathrm{L}$.
\end{abstract}

Keywords: Landfill leachate. Nitrite. Nitritation. Denitritation. Ethanol. 


\section{LISTA DE FIGURAS}

Figura 3.1 Fases de estabilização dos resíduos sólidos urbanos 13

Figura 3.2 Classificação dos diferentes tipos de isoterma de adsorção 30

Figura 3.3 Isotermas de adsorção mais comuns encontradas a partir de soluções aquosas em materiais carbonosos

Figura 3.4 Consumo de oxigênio e carbono no processo de nitrificação/desnitrificação convencional e via nitrito

Figura 3.5 Diferentes estados de oxi-redução do nitrogênio no processo de nitrificação/desnitrificação

Figura 3.6 Possível caminho metabólico para a nitrificação e desnitrificação

Figura 3.7. Curvas das velocidades máximas específicas de crescimento em função da temperatura para Nitrossomonas $\mathrm{sp} e$ Nitrobacter $\mathrm{sp}$

Figura 4.1 Vista geral do Aterro Sanitário São João

Figura 4.2 Vista superior do reator biológico R-1 durante a etapa aeróbia de um dos ciclos de tratamento

Figura 4.3 Componentes do reator biológico R-2 (a) reator de chapa de aço inoxidável; (b) agitador mecânico; (c) compressor de ar

Figura 4.4 Equipamento jar-test empregado no pós-tratamento físico-químico do efluente gerado nos reatores biológicos

Figura 4.5 Seqüencia cronológica dos ciclos de tratamento

Figura 4.6 Esquema dos pontos, frequência de amostragem e variáveis físico-químicas monitoradas (Primeira Etapa-Fase I)

Figura 4.7 Esquema dos pontos, frequência de amostragem e variáveis físico-químicas monitoradas (Primeira Etapa-Fase II)

Figura 4.8 Esquema dos pontos, frequência de amostragem e variáveis físico-químicas monitoradas durante a Segunda Etapa da pesquisa

Figura 4.9 Pontos de amostragem e variáveis físico-químicas monitoradas durante a Terceira Etapa da pesquisa (Reator de Polimento) 
Figura 4.10 Pontos de amostragem e variáveis físico-químicas monitoradas durante a Quarta Etapa da pesquisa (Reator R-2)

Figura 5.1 Box Plot: Variação das concentrações médias das formas de nitrogênio ao longo da reação anóxica (a) $\mathrm{NKT}$ (b) $\mathrm{N}_{-} \mathrm{NH}_{3}$ (c) $\mathrm{N}_{-} \mathrm{NO}_{2}{ }^{-}$(Primeira Etapa-Fase I)

Figura 5.2 Box Plot: Variação das concentrações médias das formas de nitrogênio ao longo da reação anóxica (a) NKT (b) N-NH 3 (Primeira Etapa - Fase II) 104

Figura 5.3 Box Plot: Variação das concentrações médias de $\mathrm{N}^{-\mathrm{NO}_{2}}{ }^{-}$ao longo da reação anóxica (Segunda Etapa)

Figura 5.4 Variação das concentrações médias de COT ao longo da reação anóxica (Segunda Etapa)

Figura 5.5 Concentrações médias das taxas de desnitritação (a) específicas (b) volumétricas (Segunda Etapa)

Figura 5.6 Box Plot: Variação das concentrações médias das formas de nitrogênio ao longo da reação anóxica e aerada (a) $\mathrm{NKT}$ (b) $\mathrm{N}^{-\mathrm{NH}_{3}}$ (c) $\mathrm{N}_{-} \mathrm{NO}_{2}{ }^{-}$(Primeira Etapa - Fase I)

Figura 5.7 Box Plot: Variação das concentrações médias das formas de nitrogênio ao longo da reação anóxica e aerada (a) $\mathrm{NKT}$ (b) $\mathrm{N}^{-\mathrm{NH}_{3}}$ (c) $\mathrm{N}-\mathrm{NO}_{2}{ }^{-}$(Primeira Etapa - Fase II)

Figura 5.8 Box Plot: Variação das concentrações médias de nitrito ao longo da reação anóxica e aerada (Segunda Etapa)

Figura 5.9 Quantificação de bactérias nitritantes ao longo da Segunda Etapa

Figura 5.10 Variação das concentrações de matéria orgânica ao longo da etapa aerada dos ciclos de tratamento da Segunda Etapa

Figura 5.11 Concentrações médias de COT ao longo da fase aerada da Segunda Etapa 121

Figura 5.12 Comparação da DQO (mg/L) ao final das fases anóxica e aerada dos ciclos de tratamento durante a Primeira Etapa da pesquisa

Figura 5.13 Comparação da DQO (mg/L) ao final das fases anóxica e aerada dos ciclos de tratamento durante a Segunda Etapa da pesquisa

Figura 5.14 Comparação das taxas de desnitritação (específicas e volumétricas) e a idade de lodo ao longo da etapa anóxica dos ciclos de tratamento (Reator de Polimento) 
Figura 5.15 Variação da concentração de COT ao longo da etapa anóxica e aerada dos ciclos de tratamento no Reator de Polimento (Terceira Etapa)

Figura 5.16 Box Plot: Concentrações médias de NKT durante a fase aerada da Quarta Etapa

Figura 5.17 Box Plot: Concentrações médias de $\mathrm{N}-\mathrm{NH}_{3}$ durante a fase aerada da Quarta Etapa

Figura 5.18 Box Plot: Concentrações médias de $\mathrm{N}_{-} \mathrm{NO}_{2}{ }^{-}$durante a fase aerada e anóxica da Quarta Etapa

Figura 5.19 Comparação das concentrações das formas oxidadas de nitrogênio ao longo dos ciclos de tratamento da fase aerada (Quarta Etapa)

Figura 5.20 Quantificação de bactérias nitrificantes ao longo dos ciclos de tratamento da fase aerada (Quarta Etapa)

Figura 5.21 Box Plot: Concentração de COT durante a fase aeróbia e anóxica da Quarta Etapa

Figura 5.22 Comparação das taxas de desnitritação específica e volumétrica ao fínal da reação anóxica com a idade de lodo do sistema (Quarta Etapa) 


\section{LISTA DE TABELAS}

Tabela 3.1 Classificação dos resíduos sólidos

Tabela 3.2 Componentes discriminados na composição gravimétrica típica de resíduos de origem domiciliar e comercial

Tabela 3.3 Destino final dos resíduos sólidos, por unidades de destino dos resíduos no Brasil (1989-2008) 8

Tabela 3.4 Composição do lixiviado de aterros sanitários (valores em faixa) 15

Tabela 3.5 Concentração de nitrogênio amoniacal e matéria orgânica em lixiviados 16

Tabela 3.6 Concentrações dos compostos orgânicos xenobióticos encontrados com maior frequência em lixiviados de aterros sanitários

Tabela 3.7 Classificações dos lixiviados relacionados à idade do aterro

Tabela 3.8 Evolução das variáveis em função da fase de decomposição dos resíduos depositados nas células de aterros sanitários

Tabela 3.9 Tratamento de lixiviados através do processo de coagulação - floculação

Tabela 3.10 Tratamento de lixiviados de aterro sanitário aplicando precipitação química 25

Tabela 3.11 Tratamento de lixiviados de aterro sanitário aplicando arraste com ar

Tabela 3.12 Tratamento de lixiviados de aterro sanitário aplicando processos de adsorção29

Tabela 3.13 Sistemas mais explorados para a produção de radical hidroxila

Tabela 3.14 Aplicação de processos oxidativos avançados no tratamento de lixiviados de aterro sanitário

Tabela 3.15 Tratamento de lixiviados de aterro sanitário por processos de separação por membranas

Tabela 3.16 Eficiência dos processos de tratamento físicos e físico-químicos em função da idade do lixiviados de aterro sanitário $\quad 44$

Tabela 3.17 Recirculação do lixiviados as células do aterro sanitário

Tabela 3.18 Tratamento combinado de lixiviado de aterro sanitário e esgoto doméstico 
Tabela 3.19 Lodos ativados no tratamento de lixiviados de aterro sanitário

Tabela 3.20 Lagoas aeradas no tratamento de lixiviados de aterro sanitário

Tabela 3.21 Filtros percoladores no tratamento de lixiviados de aterro sanitário

Tabela 3.22 Tratamento de lixiviados de aterro sanitário por processos anaeróbios

Tabela 3.23 Trabalhos visando à remoção de $\mathrm{N}-\mathrm{NH}_{3}$ pelo processo de nitritação de águas residuais

Tabela 3.24 Padrões de qualidade máximos admissíveis das formas de nitrogênio para cursos de água

Tabela 4.1 Caracterização do lixiviado produzido no Aterro Sanitário São João

Tabela 4.2 Condições Operacionais - Primeira Etapa da pesquisa

Tabela 4.3 Condições Operacionais - Segunda Etapa da pesquisa

Tabela 4.4 Condições Operacionais - Terceira Etapa da pesquisa (Reator de Polimento) 89

Tabela 4.5 Condições Operacionais - Quarta Etapa da pesquisa (Reator R-2)

Tabela 4.6 Condições Operacionais aplicadas no pós-tratamento físico-químico do efluente do reator biológico - Variação da dosagem de sal de ferro e do $\mathrm{pH}$ (Fase I)

Tabela 4.7 Condições Operacionais aplicadas no pós-tratamento físico-químico do efluente do reator biológico - Adição de CAP (Fase II) 94

Tabela 4.8 Condições Operac. aplicadas no estudo da cinética de adsorção do CAP 95

Tabela 4.9 Relação das variáveis e métodos utilizados

Tabela 5.1. Concentração de metais no lixiviado empregado na pesquisa

Tabela 5.2. Resultados da Fase Anóxica dos ciclos de remoção - Primeira Etapa

Tabela 5.3. Resultado da Fase Anóxica dos ciclos de remoção - Segunda Etapa

Tabela 5.4. Resultado da Fase Aeróbia dos ciclos de remoção - Primeira Etapa

Tabela 5.5. Resultado da Fase Aeróbia dos ciclos de remoção - Segunda Etapa

Tabela 5.6. Concentração da $\mathrm{DQO}(\mathrm{mg} / \mathrm{L})$ nas diferentes etapas do tratamento

Tabela 5.7. Resultados dos ciclos de remoção no Reator de Polimento - Terceira Etapa 127 
Tabela 5.8. Resultado da Fase Aeróbia dos ciclos de remoção - Quarta Etapa

Tabela 5.9. Resultado da Fase Anóxica dos ciclos de remoção - Quarta Etapa

Tabela 5.10. Concentração de metais no sobrenadante dos Reatores R-1 e R-2 após a finalização do processo de tratamento biológico

Tabela 5.11. Concentração de metais no lodo de alíquotas retiradas dos Reatores R-1 e R-2

Tabela 5.12. Resultados do pós-tratamento físico-químico do efluente do reator biológico Variação da dosagem de sal de ferro, $\mathrm{pH}$ e do gradiente médio de velocidade (Fase I - Quinta Etapa)

Tabela 5.13. Resultados do pós-tratamento físico-químico do efluente do reator biológico Adição de CAP (Fase II)

Tabela 5.14. Resultados do estudo da cinética de adsorção do CAP

Tabela 5.15. Resultados dos testes de toxicidade aguda 


\section{INDICE}

1. INTRODUÇÃO

2. OBJETIVOS

4

2.1. Objetivo Geral

4

2.2. Objetivos Específicos

3. REVISÃO DA LITERATURA 5

3.1. Resíduos sólidos urbanos (RSU) 5

3.2. Aterro Sanitário 8

3.3. Processo de degradação de RSU e geração de lixiviados em aterros sanitários $\quad 10$

3.4. Lixiviado de aterro sanitário $\quad 13$

3.4.1. Composição do lixiviado formado em aterro sanitário 14

3.4.2. Classificação do lixiviado de Aterro Sanitário 18

3.5. Tratamento de lixiviado de aterro sanitário 20

3.5.1. Sistemas de tratamento fundamentados em métodos físicos e físico-químicos 20

3.5.1.1. Coagulação/Floculação/Sedimentação 21

3.5.1.2. Precipitação química 23

3.5.1.3. Arraste com ar (stripping) 25

3.5.1.4. Adsorção 28

3.5.1.5. Oxidação química 33

3.5.1.6. Tratamento Eletroquímico 38

3.5.1.7. Membranas $\quad 38$

3.5.1.9. Evaporação $\quad 42$

3.5.2. Sistemas de tratamento fundamentados em processos biológicos 45

3.5.2.1. Recirculação do lixiviado no aterro sanitário 45

3.5.2.2. Tratamento combinado com esgoto 47

3.5.2.3. Lagoas de estabilização

3.5.2.4. Processos biológicos aeróbios convencionais 52

3.5.2.4.1. Lodos ativados 53

$\begin{array}{ll}\text { 3.5.2.4.2. Lagoas aeradas } & 54\end{array}$ 
3.5.2.4.4. Biodiscos 57

3.5.2.5. Processos biológicos anaeróbios 58

3.5.2.6. Tratamento biológico de lixiviado por processo de Nitritação/Desnitrtação 61

3.6. Legislação pertinente aos lixiviados $\quad 72$

4. MATERIAL E METODOS 74

$\begin{array}{lll}\text { 4.1. Descrição geral da pesquisa } & 74\end{array}$

4.1.1. Caracterização do lixiviado de aterro sanitário utilizado durante a pesquisa 75

4.1.2. Inoculo e partida do sistema

4.1.3. Instalações experimentais e procedimentos de rotina 77

4.2. Operação e condução da pesquisa $\quad 79$

4.2.1. Primeira Etapa: Estudos Preliminares e ajuste das condições operacionais no Reator R$\begin{array}{ll}1 & 79\end{array}$

4.2.2. Segunda Etapa: Estudo da eficiência do processo de nitritação/desnitritação 84

4.2.3. Terceira Etapa: Reator de Polimento 87

4.2.4. Quarta Etapa: Estudo do processo de nitritação/desnitritação empregando uma fonte $\begin{array}{ll}\text { externa de carbono (etanol) } & 90\end{array}$

4.2.5. Quinta Etapa: Pós-tratamento físico-químico 93

4.3. Parâmetros físico-químicos e microbiológicos 96

5. APRESENTACÃO E DISCUSSÃO DOS RESULTADOS 99

5.1. Caracterização do lixiviado empregado na pesquisa 99

5.2. Estudo da eficiência do processo de nitritação/desnitritação no Reator R-1. Primeira e $\begin{array}{ll}\text { Segunda Etapa } & 102\end{array}$

5.2.1. Desnitritação biológica aplicando a matéria orgânica do lixiviado como fonte de carbono

5.2.2. Estudo do Processo Nitritação: Condições para o acúmulo de nitrito. Primeira e Segunda Etapa.

5.3. Terceira Etapa: Tratamento complementar adicionando etanol como fonte de carbono para a desnitritação - Reator de Polimento 
5.4. Quarta Etapa: Estudo do processo de nitritação/desnitritação empregando etanol como fonte externa de carbono 128

5.5. Quinta Etapa: Pós-tratamento físico-químico 138

6. CONCLUSÕES

7. REFERÊNCIAS BIBLIOGRÁFICAS

8. APENDICE 